\title{
MULTI-PORT SOLUTION FOR EFFICIENT, LOW-COST OPTICAL SWITCHES
}

\author{
Devin R. Berg \\ Department of Mechanical Engineering \\ University of Minnesota - Twin Cities \\ Minneapolis, MN, USA
}




\section{ABSTRACT}

Optical switches offer a promising solution for routing optical-fiber based signals which are popular within the telecommunications industry. The project presented here investigated the possibility of utilizing a large displacement electrostatic comb-drive actuator to permit a single input signal to be routed to one of multiple output optical ports. A review of the literature revealed several solution possibilities that have been explored in the past; however, the majority of the multiport solutions use complicated actuator arrays to achieve the necessary routing capability. By using a single degree of freedom system, the complexity of both the manufacturing process as well as the control method for the final system can be simplified. Presented here is a novel design for an optical switch including the fabrication process, mask layout, and packaging method necessary to realize this design as a real world application.

\section{PROBLEM DEFINITION AND RATIONALE}

Optical switches are becoming increasingly necessary as various telecommunication industries transition to optical-fiber based communication networks. Many of the traditional switch designs in this field require a conversion of the optical signal to an electrical signal for routing and then back to an optical signal for transmission, which is subject to changes in the data rate and protocol. Therefore, the development of a low-cost, reliable multi-port optical switch would be a beneficial technology for the telecommunication industry [1-3].

This project will require the investigation and development of both a method of redirecting an input optical signal to one of several outputs as well as a method of manufacturing the optical switch design, which will be capable of mass production at low cost.

The characteristics that are desirable in modern optical switches include high ON/OFF contrast ratio, low crosstalk between outputs, fast switching speed, efficient operation, and compact design [2]. There are a variety of approaches to this problem which have been explored previously in the literature. Possible solutions include moving the input optical fiber in order to direct it towards the appropriate output or perhaps reflecting the input signal to the appropriate output through the use of mirrors. In either solution space it is likely that some form of linear actuation will be necessary and therefore part of the project will involve an exploration of the various actuation methods such as piezoelectric, comb-drive structures, and thermally activated materials.

Once a device design has been chosen, it will be necessary to develop a fabrication process that avoids high cost techniques if possible in order to maintain a low fabrication cost. Possible fabrication techniques include material deposition, bulk micro-machining, and surface micromachining, among others.

There are many possible design configurations for this device and this project will require a selection of the final design through a balance of device performance and fabrication cost.

\section{LITERATURE REVIEW}

Optical switches are among the growing list of technologies undergoing rapid miniaturization. This advancement is fueled by the telecommunications industry transitioning to all-optical networks thus increasing the demand for high volume, low cost, efficient optical switches [1]. In order to achieve these goals, MEMS technology has been identified as an attractive option. Past research has explored novel MEMS devices in order to overcome some of the limitations such as higher port counts, cross-talk, and reliability [4]. The application of MEMS technology to this application has taken the form of either 2D or 3D configurations (See Fig. 1) in which 2D MEMS switches are simple to operate but have lower port counts while 3D MEMS switches are able to include a greater number of ports but require complex and expensive control systems for proper operation [5]. Several mechanisms have been pursued in the literature including thermal expansion actuators, gap-closing electrostatic actuators, and comb drive actuators $[2,4]$. Each of these methods has been shown to have advantages and disadvantages. Recent advancement in the area of MEMS optical switches have included the use of deformable micromirrors, multi-actuated two dimensional mirror arrays, and three-dimensional, two degree of freedom, analog mirror arrays. While each of these solutions provides beneficial characteristics, there remains room for improvement.

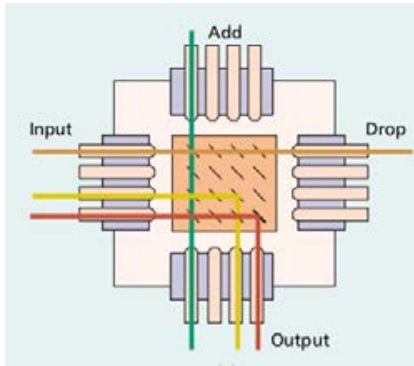

(a)

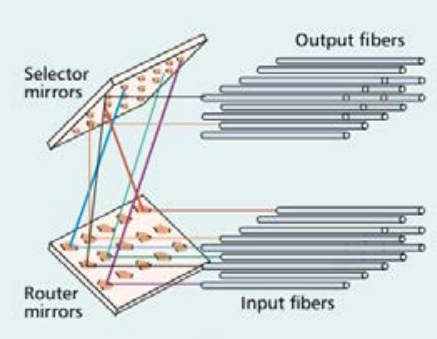

(b)
Figure 1: Examples of 2D (a) and 3D (b) MEMS optical switch designs [5].

After reviewing existing technologies in the area of MEMS optical switching, there remains room for the development of a low-cost, multi-port, simple MEMS solution. Much recent research has been performed in the area of increasing the displacement range of linear actuators [6-8]. This advancement provides the ground work for revisiting the use of linear actuators for simple multi-port optical switches. A largedisplacement actuator may be useful in achieving multi-port switching with one degree of freedom. This technique would minimize the complexity of the control architecture and increase device reliability. Electrostatic comb-drive linear actuators appear to be the most advantageous technology for achieving reliable large-displacement actuation; however, other actuation techniques will continue to be considered as well. Much of the design in this project will be focused on achieving 
large-displacements as well as taking advantage of that displacement range to provide access to a greater number of optical ports with a one degree of freedom device.

\section{POTENTIAL SOLUTIONS}

For a multi-port, efficient, low-cost optical switch, the critical characteristics that are desired from the mechanism include efficiency, high switching speed, mechanical reliability, compactness, and cost/manufacturability. Therefore, some potential solutions have been generated and then ranked based upon these criteria. Variables affecting the potential solution space for the present device include the principle of mechanism motion, method for achieving that motion, and the mechanism for routing the input signal to the appropriate output signal.

Designs A through C employ vertical deflection of a cantilever beam as the method for achieving motion. This arrangement uses vertical micromirrors attached to a cantilever beam using microhinges. An array of these beams would permit the use of several optical output paths. The mirror could then be actuated into and out of the path of the optical signal through beam deflection using actuation techniques such as electrostatic actuation (Design A), piezoelectric actuation (Design B), or thermal bimetallic actuation (Design C). These designs would perform fairly well in the areas of efficiency and speed; however, due to the complexity of manufacturing a hinged vertical micromirror, the cost of fabrication would likely be greater than other proposed designs. The thermal bimetallic method would likely have a slower response time than the other two methods due to its dependence on the heating and cooling rate of the material. Additionally, for all three of these designs, each output would require an individual cantilever beam actuator and therefore the size of the mechanism per output channel would likely be larger than other designs.

Design D uses a different actuation method than Designs A through $\mathrm{C}$. This design would achieve actuation through the use of an electrostatic comb drive actuator. Comb drive actuators have been used previously in the design of optical switches and have characteristics which allow them to achieve large displacements for relatively low voltage differentials [9]. A drawback of this design is the large size of each individual comb drive actuator; however, since one actuator could potentially route the optical signal to multiple output channels, this characteristic would likely not be an issue. Additionally, the fabrication of this mechanism could likely be achieved in fewer steps than some of the other design solutions.

Designs $\mathrm{E}$ and $\mathrm{G}$ achieve actuation through a slight variation on the method used for Designs A through C. These designs use horizontal deflection of a cantilever beam in order to move a mirror into and out of the path of the optical signal. This method of actuation has the benefit of simplicity over Designs A through $\mathrm{C}$ since it would not be necessary to fabricate a hinged vertical micromirror. Instead, the cantilever beam itself could be coated with a reflective material such as gold in order to form the mirror surface. Additionally, since the mirror would progress through a range of angles with respect to the input channel is it possible for a single actuator to provide redirection of the optical signal to multiple output channels. Each of these designs is differentiated by the actuation method used similar to Designs A through C, these actuation techniques include electrostatic actuation (Design E), piezoelectric actuation (Design F), or thermal bimetallic actuation (Design $\mathrm{G)}$.

Summarized in Table 1, a comparison of each of the potential design solutions suggests that Design $\mathrm{D}$ would be the most optimal of the potential solutions. Design D uses a electrostatic comb drive actuator to achieve mechanism motion, which has been used previously as a reliable option for optical switch design. The primary disadvantages to Designs A through $\mathrm{C}$ are the complexity of fabrication of the hinged vertical micromirror. This disadvantage is addressed in Designs D through $G$ where the mirror surface(s) could be fabricated directly on existing structure. Design E represents the nearest competition for Design D, however, it has slight perceived disadvantages in terms of reliability and manufacturability due to the dependence of the design on precise positioning of the cantilever beam in deflection. Therefore, Design D was selected as the solution for the present project. 
Table 1: Decision matrix comparing each of the potential design solutions.

\begin{tabular}{|c|c|c|c|c|c|c|}
\hline & Efficiency & Speed & Reliability & Compactness & Cost/Manufacturability & RANK \\
\hline $\begin{array}{l}\text { Weighting } \\
\text { Factor }\end{array}$ & 0.20 & 0.10 & 0.15 & 0.20 & 0.35 & 1.00 \\
\hline \multirow{2}{*}{ Design A } & 8 & 8 & 5 & 5 & 3 & \multirow{2}{*}{5.20} \\
\hline & 1.60 & 0.80 & 0.75 & 1.00 & 1.05 & \\
\hline \multirow{2}{*}{ Design B } & 5 & 8 & 5 & 5 & 3 & \multirow{2}{*}{4.60} \\
\hline & 1.00 & 0.80 & 0.75 & 1.00 & 1.05 & \\
\hline \multirow{2}{*}{ Design C } & 6 & 4 & 5 & 5 & 3 & \multirow{2}{*}{4.40} \\
\hline & 1.20 & 0.40 & 0.75 & 1.00 & 1.05 & \\
\hline \multirow{2}{*}{ Design D } & 8 & 8 & 7 & 7 & 8 & \multirow{2}{*}{7.65} \\
\hline & 1.60 & 0.80 & 1.05 & 1.40 & 2.80 & \\
\hline \multirow{2}{*}{ Design E } & 8 & 8 & 6 & 8 & 7 & \multirow{2}{*}{7.35} \\
\hline & 1.60 & 0.80 & 0.90 & 1.60 & 2.45 & \\
\hline \multirow{2}{*}{ Design F } & 6 & 8 & 6 & 8 & 6 & \multirow{2}{*}{6.60} \\
\hline & 1.20 & 0.80 & 0.90 & 1.60 & 2.10 & \\
\hline \multirow{2}{*}{ Design G } & 6 & 4 & 6 & 8 & 6 & \multirow{2}{*}{6.20} \\
\hline & 1.20 & 0.40 & 0.90 & 1.60 & 2.10 & \\
\hline
\end{tabular}

\section{CUSTOMER REQUIREMENTS AND SPECIFICATIONS}

The goal of this project is to propose a novel solution to a multi-port optical switch, which operates efficiently, has low fabrication costs, and enables the routing of a single input channel to a variety of output channels. In this section, design specifications will be described based on the checklist provided.

1. Functional requirements

a. Overall geometry: The proposed device needs to be constrained in overall size in order to permit the use of multiple switches in an array configuration. However, the desired output range of motion from the actuator places certain limitations on the minimum size allowed. These dimensions will be on the order of 1000 to 2000 microns in each direction.

b. Forces involved: For the device to operate, the force generation mechanism must be capable of overcoming the spring force for the amount of deflection desired. Spring force is defined by the beam bending characteristics of the suspension structure. Similarly the force produced is defined by the properties of the actuation method such as resistivity and geometric properties of the electrostatic comb drive actuator.

2. Safety requirement

a. Operational: Since the optical switch is driven through the application of a voltage, it is necessary to ensure that the switch is not accessible during normal operation in order to prevent accidental electrocution.

3. Quality requirement a. Reliability: To ensure reliable operation, the device must be sealed off from the environment in order to prevent contamination from dust and debris which may inhibit its ability to operate as designed.

b. Quality assurance: Quality of the device can be measured by comparing the optical strength of the input signal to that of the output signal. A high quality optical switch has low signal loss as the signal is transmitted from the input to the appropriate output. Additionally, there much be minimal crosstalk between output channels.

4. Manufacturing requirement

a. Production of components: Mass fabrication will be carried out through the use of MEMS surface micro-machining techniques on silicon wafers. Each wafer can be used to manufacture multiple individual units, where the total number depends on the overall size of the wafer used.

b. Assembly: As previously mentioned, the device must be sealed from the environment and therefore the packaging of the device should be high quality to prevent any unintended contaminants. Additionally, the packaging should be designed such that multiple devices can be arranged in an array with little wasted space.

5. Timing requirement

a. Schedule: The complicated nature of this sort of electrostatic actuator requires multiple photolithography processes and therefore the fabrication schedule will be slightly longer when compared to other simpler designs. 
6. Economic requirement

a. Marketing analysis: Optical switches are a popular option in the world of telecommunications and as the market grows further into developing countries and previously untapped rural areas, the need for innovative, cost-effective optical switches will increase.

b. Cost: Since the production of these optical switches is based on the MEMS techniques and given the high volume production necessary to meet growing demand, these devices can be manufactured in mass therefore reducing the cost of each individual unit.

7. Ergonomic requirement

a. User needs: The proposed optical switch is intended to be a simple plug-in solution for the telecommunications industry. Therefore, the device must have a simple method of installation by a reasonably knowledgeable technician.

8. Ecological requirement

a. Material selection: The device may be exposed to a variety of environmental conditions depending upon the installation location. The materials used should be compatible with variations in temperature, vibration, and possible UV exposure. This is especially true for the components used in the packaging of the device.

9. Aesthetic

a. Future expectation: As the complexity of the telecommunications industry increases, the demand for greater complexity in optical switches increases as well. It will be required for the optical switches to route signals between a greater number of inputs and outputs and to do it quicker and with more efficiency.

10. Life-cycle

a. Maintenance: Since this device is likely to often be deployed to locations where regular servicing may be cost prohibitive, the device should require minimal maintenance for reliable operation. Further, the device should allow for quick installation and removal in the event that it should fail.

b. Disposal: Since this device does not interact with any hazardous contaminants, it may be possible for the device to be recycled if it is financially prudent to do so.

\section{MODELING AND SCALING EFFECTS}

One of the key components of the design for a novel optical switch is the method for achieving large displacements. In the present design, the method chosen for producing the mechanism displacement is use of an electrostatic comb-drive actuator. The comb-drive actuator achieves motion through the application of a voltage difference across fixed and movable interdigitated comb fingers. This voltage difference produces an electrostatic field between the neighboring fingers and thus induces an attractive force that can be modeled as a parallel plate arrangement, see Figure 2 [10].

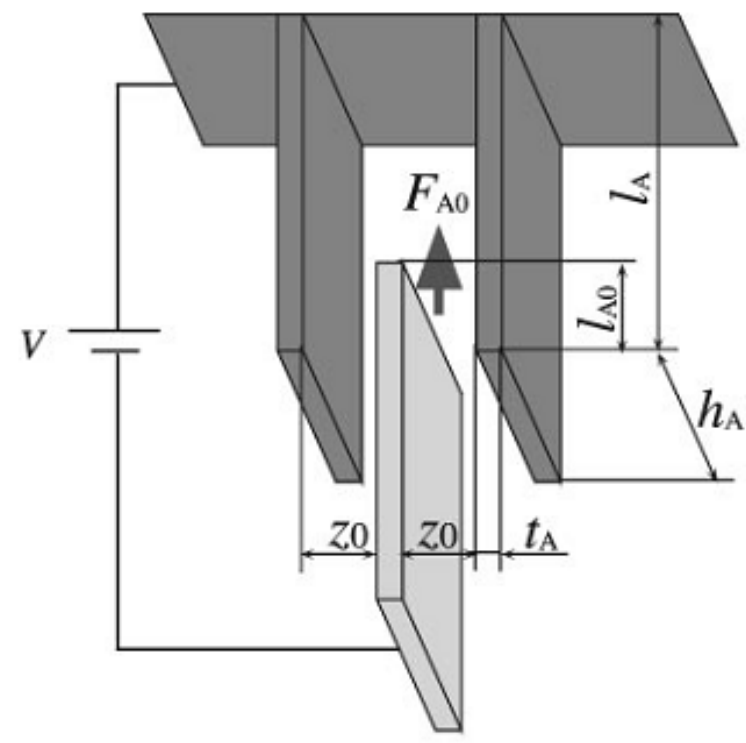

Figure 2: Geometry of a single set of comb-drive fingers [11].

Using this parallel plate model, with the geometry shown in Figure 1, the capacitance of parallel plates can be expressed as,

$$
C=\frac{2 n \varepsilon_{0} h_{A} l_{A 0}}{z_{0}}
$$

where $n$ is the number of fingers and $\varepsilon_{0}$ is dielectric constant in free air. From this, the force, $F_{A 0}$, can be described as,

$$
F_{A 0}=\frac{1}{2} \frac{\delta C}{\delta y} V^{2}=\frac{n \varepsilon_{0} h_{A}}{z_{0}} V^{2}[10,11] .
$$

Concurrent to this, it is necessary to model the effect of the spring used to return the actuator to its initial position when the voltage difference across the comb drive fingers is removed. One design for the spring suspension system is the folded flexure design shown in Figure 3. 


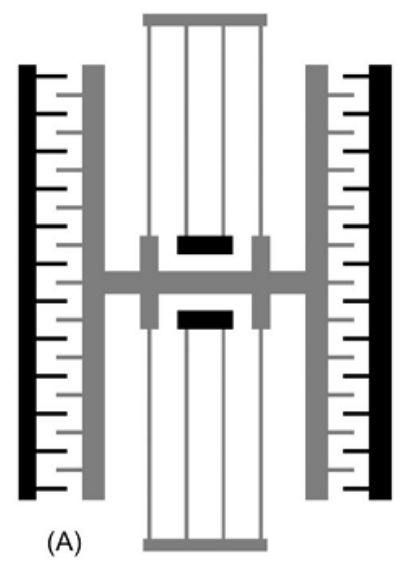

Figure 3: Folded flexure design commonly used for large displacement electrostatic comb drive actuators [7].

The force that must be applied to the spring to achieve the desired deflection can be computed by modeling the individual springs as beam elements with fixed ends. Then the force of the spring is modeled as,

$$
F_{\text {spring }}=\delta \frac{24 E I}{l^{3}}=2 \delta \frac{E h_{A} t_{S}^{3}}{l^{3}} \text { [12]. }
$$

By setting the actuator force equal to the spring force, it is possible to design the electrostatic actuator such that the desired displacement can be achieved. The parameters that are able to be designed are the number of fingers, $n$, the spring length, $l$, the spring width, $t_{S}$, the structural thickness, $h_{A}$, the finger gap, $z_{0}$, and the operating voltage, $V$.

The scaling effects can be particularly significant and thus should be considered during the design process. For example, the force necessary to drive a fixed-fixed beam spring as proposed in the present project is greatly subjective to the effects of geometric scaling. As the overall size of the beam suspension system is scaled down by an order of magnitude from the original size, the force necessary to achieve the new scaled displacement reduced by two orders of magnitude, as seen in Figure 4.

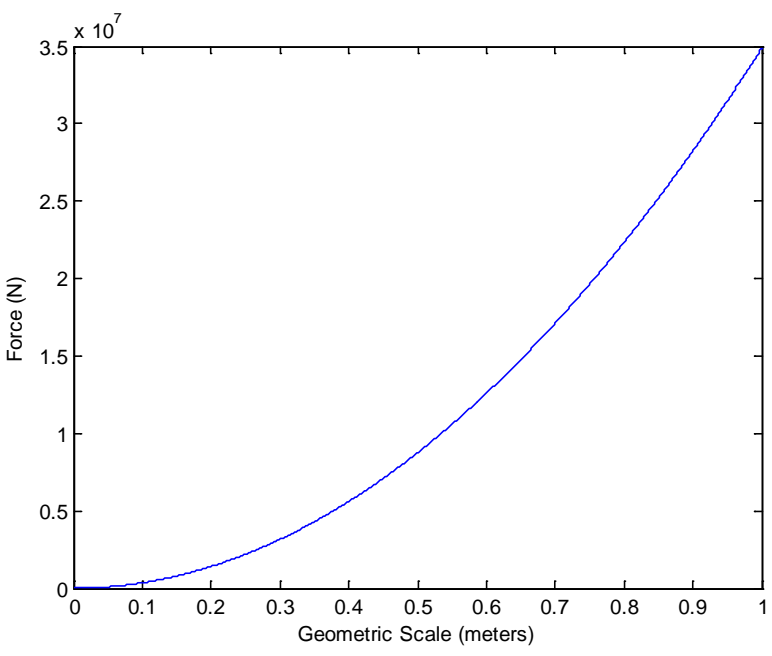

Figure 4: Effect of scale on spring suspension driving force.
This effect may be beneficial since it may be possible for the electrostatic actuator requirement to be greatly reduced for even small changes in the suspension system size. One possible realization of this is in terms of the driving voltage for the electrostatic actuator. Assuming consistent geometry of the actuator, a smaller requirement in force would mean that the driving voltage could be reduced thus resulting in a more efficient device. Additionally, it is interesting to note that the force output from the actuator is independent of geometry scaling assuming that the finger gap scales proportionally to structural thickness of the actuator fingers. Therefore, the limitation of geometric scaling of the actuator is only limited by the constraints of the fabrication process used to produce the device.

\section{FABRICATION FLOWCHART}

A possible fabrication process is presented in the following flowchart. An alternate fabrication process is presented in the literature which has been shown to produce high-aspect-ratio comb-drive structures [13]. To accompany each of the steps, a cross sectional model of the wafer throughout the fabrication process is presented alongside each step. Specific information regarding specific deposition/etch rates and recipes to be used has been adapted from the website of the Nanofabrication Center at the University of Minnesota - Twin Cities [14]. For convenience, a key correlating the different cross-hatching patterns used to the appropriate material is shown below.

Silicon Wafer

Silicon Nitride

\section{Silicon Dioxide}

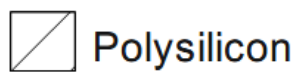

\section{Gold}

1. Start with a bare $<100>$ silicon wafer.

a. Wafer should be cleaned using the RCA clean procedure.

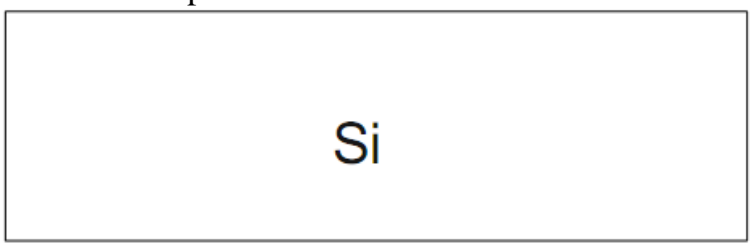

2. Deposit $2000 \AA$ of Silicon Nitride by LPCVD.

a. Deposition for 60 min. using the default Low Stress Nitride recipe.

\section{$\mathrm{Si}$}


3. Deposit $1 \mu \mathrm{m}$ of Silicon Dioxide by LPCVD.

a. Deposition for 125 min. using the PSG recipe.

b. Anneal the wafer.

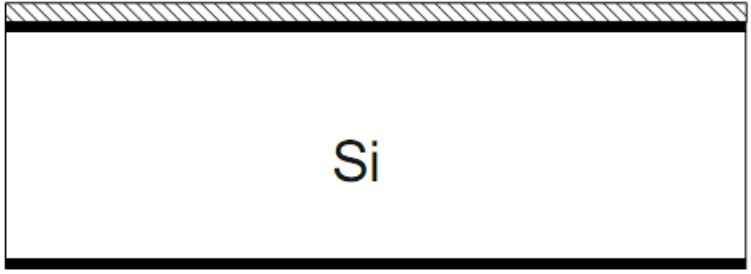

4. Perform photolithography on Silicon Dioxide.

a. Deposit photoresist (PR 1813) by spinning.

b. Expose photoresist to UV using mask \#1 (dark field).

c. Etch photoresist using photoresist developer.

d. Etch unprotected Silicon Dioxide using HF etch.

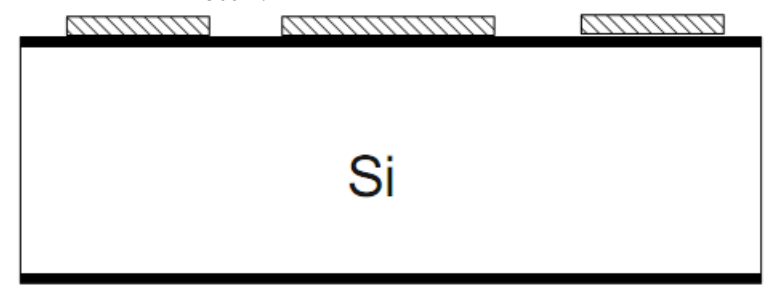

5. Deposit Polysilicon by LPCVD.

a. Deposition for 7 hours using a combined doped/undoped Polysilicon recipe.

b. Anneal the wafer to reduce the chance of undesired deformation.

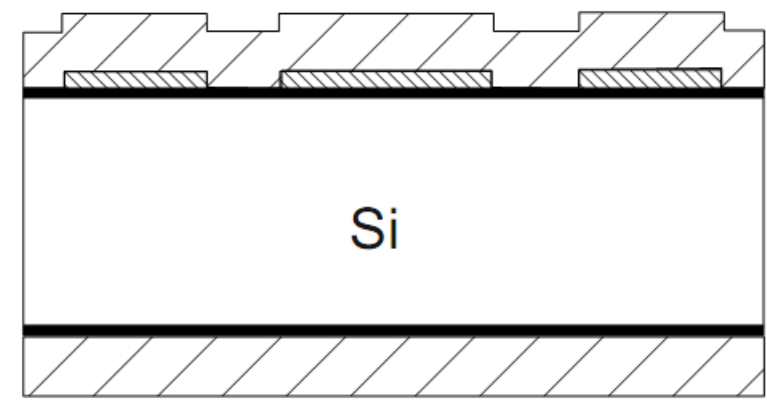

6. Perform photolithography on Polysilicon.

a. Deposit photoresist (PR 1813) by spinning.

b. Expose photoresist to UV using mask \#2 (light field).

c. Etch photoresist using photoresist developer.

d. Etch unprotected Polysilicon using RIE.

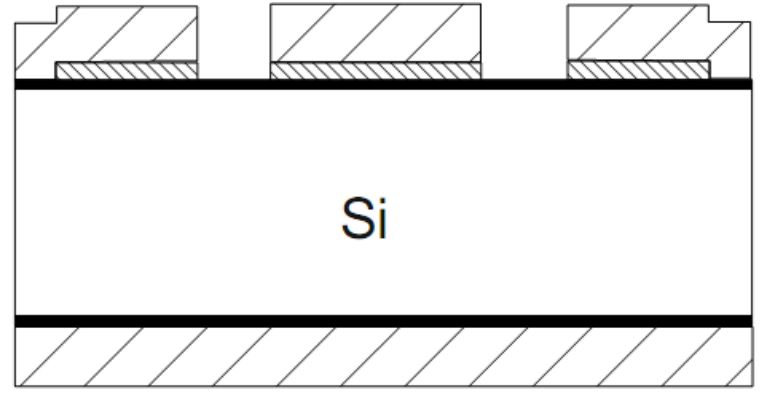

7. Deposition of a reflective gold layer by electron-beam evaporation.

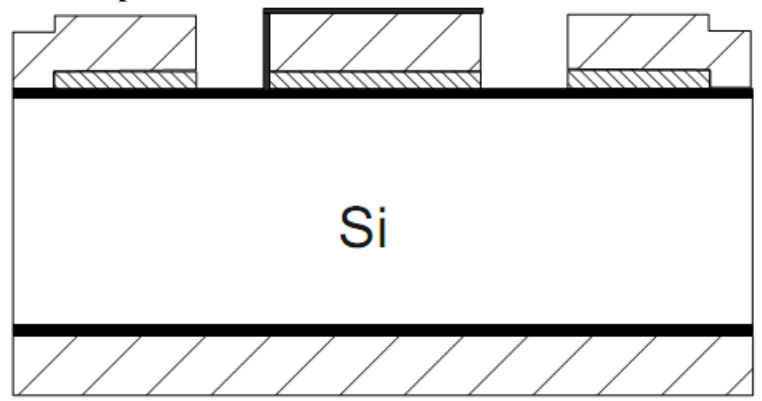

8. Remove sacrificial Silicon Dioxide by HF etch.

a. Wafer will need to go into the Critical Point Dryer to remove any remaining etchants.

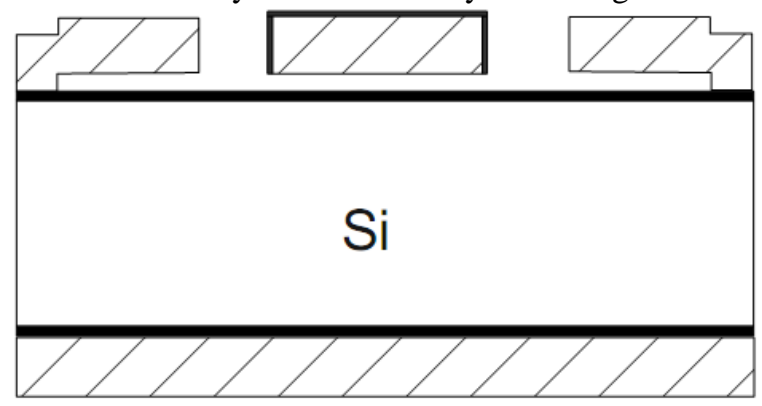

9. Wafer diced into individual units and assembled with packaging to form final product.

\section{MASK LAYOUT}

For the proposed fabrication process, there are two masks required, one for each of the photolithography steps. The first photolithography process creates the anchor locations for the polysilicon structural layer by removing portions of the sacrificial silicon dioxide layer. The mask designed to define the anchor location, as well as the necessary alignment mark, is a dark field mask, seen in Figure 5. The gray areas in the figure represent the areas that will be etched away from the silicon dioxide layer [15]. 

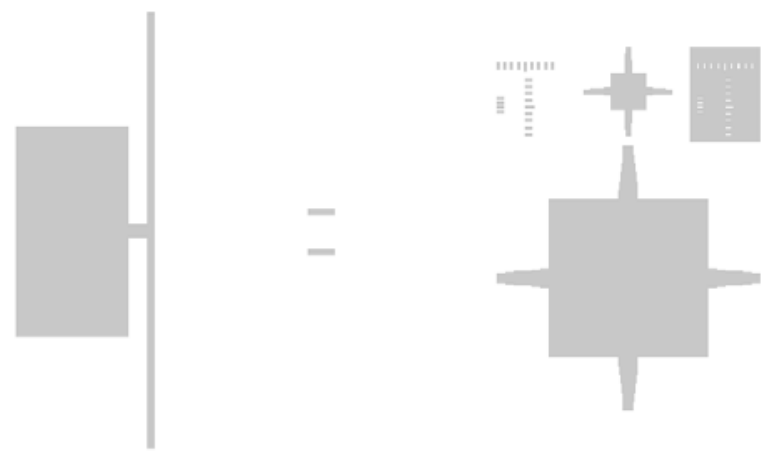

Figure 5: Mask layout to define the anchor locations.

The second photolithography process defines the polysilicon structural layer by removing the undesired portions of the structural polysilicon. The mask designed to define the structural layer is a light field mask, seen in Figure 6. The gray areas represent the areas that will remain after the polysilicon layer is etched [15]. This mask layout shows an electrostatic actuator design intended to increase the viable range of actuator displacement by reducing the influence of side instability, similar to the design described by Grade et al. [7]. Side instability is an important consideration as it is often the limiting factor in terms of how large of a displacement will be possible from a given comb-drive actuator [16-18].

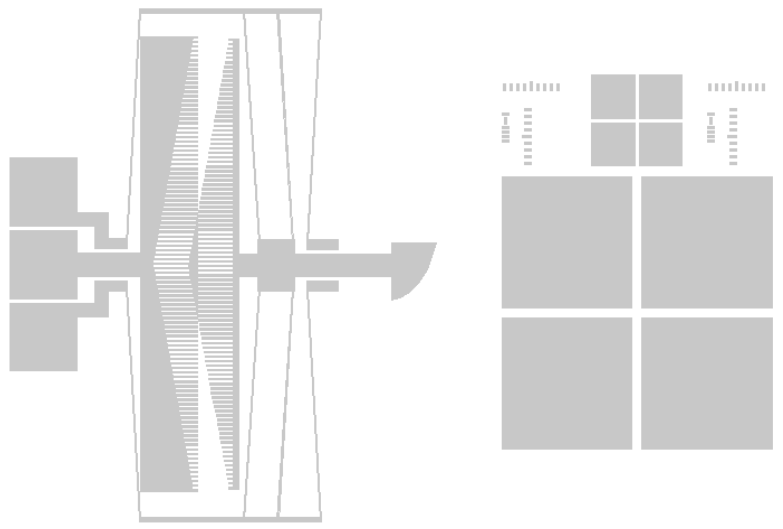

Figure 6: Mask layout to define the structure of the mechanism.

\section{PACKAGING AND TESTING}

Packaging of the proposed optical switch device has some important consideration such as protection from the environment, inclusion of an electrical interface for the control signal, mounting and securing of the device chip, securing of the input and output optical signals, and ability to use the device in an array.

In order to protect the device from external interference, the device chip needs to be placed in a sealed container that blocks the infiltration of dust and debris. The container also needs to electrically isolate the chip except for the intended electrical connections. Therefore, the chip needs to be placed on insulating stand-offs, as seen in Figure 7. The electrical connections should carry the signal to the external side of the container to be connected with the control signal input.

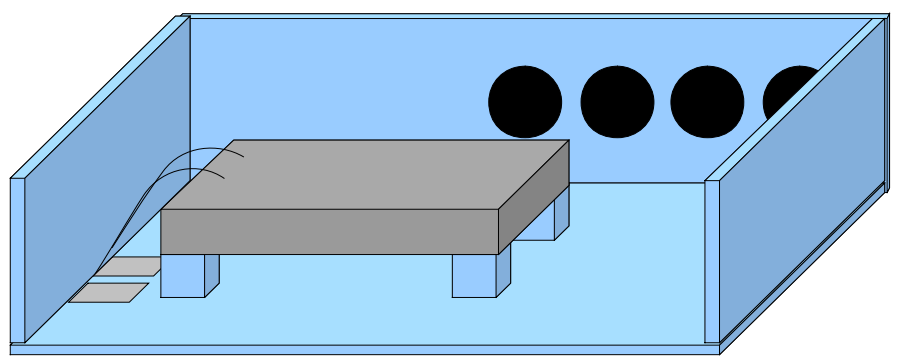

Figure 7: Internal view of proposed packaging container.

Another major consideration is the securing of the chip as well as the optical signal inputs and outputs. Since the chip contains free floating mechanical components, it is important that the chip is secured within the container and that the container is handled carefully. Additionally, the side walls of the container should include adapters to easily connect the optical signals, Figure 8.

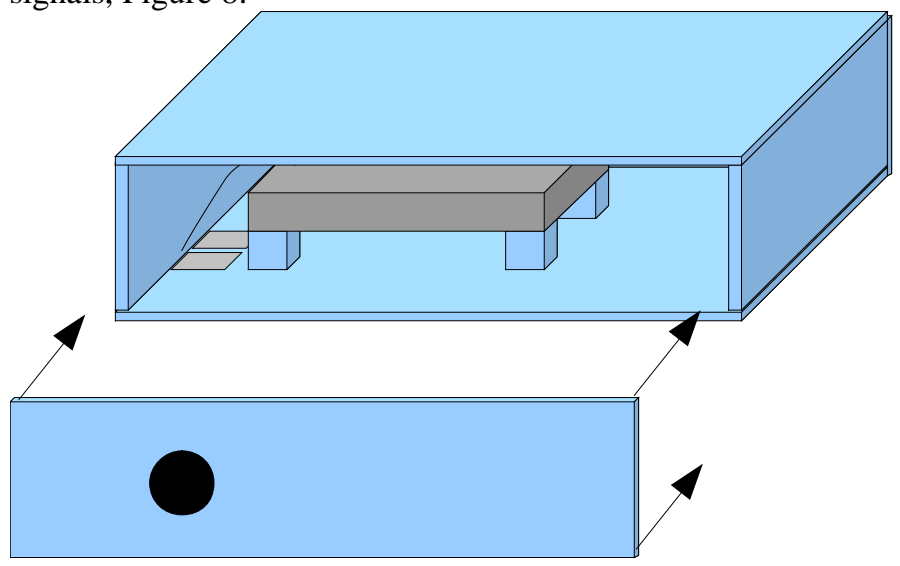

Figure 8: Sealing of the container and location of port for input optical signal.

Finally, it is desirable for the assembled unit to be easily placed into an array such that as many inputs as necessary for the application can be routed to their appropriate outputs. This is achieved by using a box shaped packaging and ensuring that the input and output optical signal connections are placed on the front and back of the unit, permitting array expansion in both the vertical and horizontal directions, seen in Figure 9. 


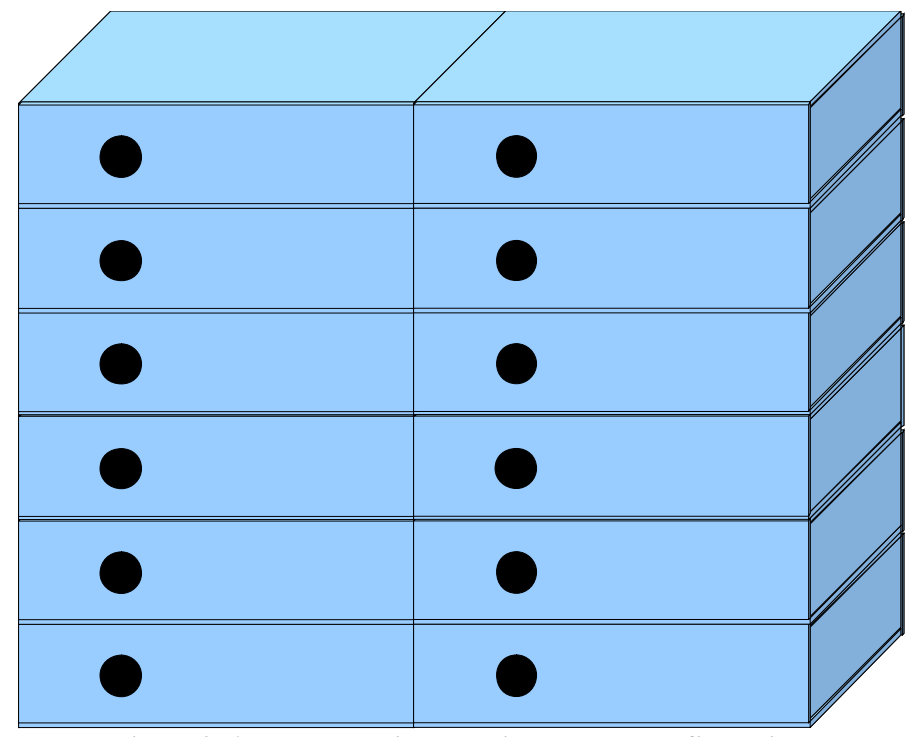

Figure 9: Assembled unit shown in an array configuration.

In order to ensure that the proposed device operates as intended, it is necessary to test its operation under simulated conditions. Characteristics that need to be evaluated include the reflectivity of the device as well as the frequency with which the signal can be routed between the different outputs [19].

The reflectivity of the device can be assessed by inputting an optical signal and measuring the intensity of the reflected optical signal. Similar optical switching applications have demonstrated that a reflectivity of $85 \%$ is more than adequate.

The response frequency of the optical switch can be evaluated by measuring the signal from the outputs as the switch is cycled through the full displacement range. The driving voltage would be applied in a sine wave and the frequency increased until the switch no longer routes the signal accurately.

\section{CONCLUSIONS}

A large-displacement electrostatic comb drive driven optical switch has been designed to address the need for a lowcost, efficient multi-port switch. The implementation method used was selected based upon an optimization for efficiency, speed, reliability, compactness, and cost/manufacturability. The presented design achieves large displacement through the use of prebent suspension beams and linearly engaging comb teeth. The switch can then be controlled by applying an analog driving voltage to adjust the displacement of the vertical micro-mirrors. The mirror array then directs the input signal to the appropriate output optical port. This can be seen in the simulated output presented in Figure 10, which shows the achieved displacement plotted against the input voltage signal for an example switch design. Additionally, it can be seen that the design with linearly engaging teeth requires a higher voltage to achieve the same displacement output for smaller displacements but this is acceptable since the design is less susceptible to side instability [7]. The code for this simulation is found in Annex A.

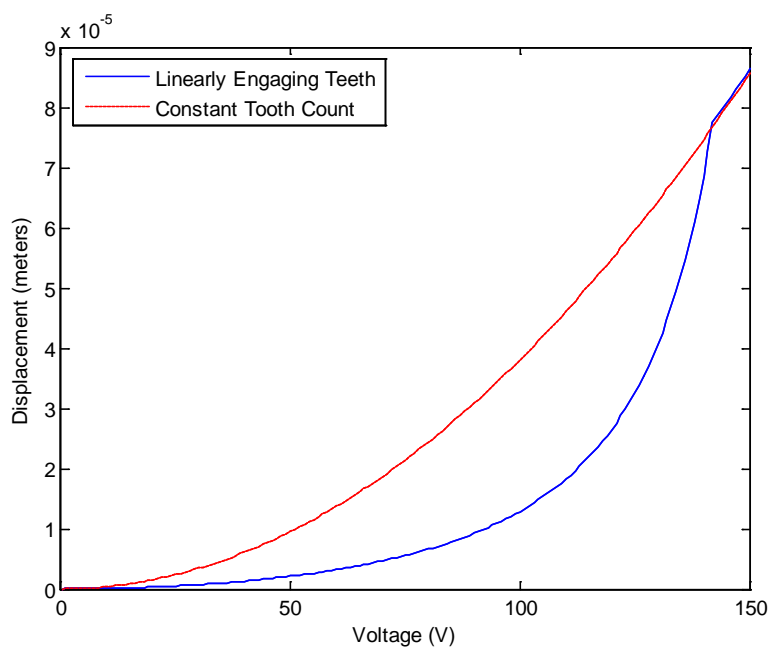

Figure 10: Simulation of output displacement versus input voltage signal.

The fabrication process to produce the designed optical switch requires only two photolithography processes and therefore should be relatively low cost for high-volume operations. Further, the small footprint of the device means that a large number of switches can be manufactured on each wafer. Finally, through the use of a simple packaging method, multiple devices can be arranged in an array to allow multiple inputs to be routed.

\section{REFERENCES}

[1] Chen, R.T., Nguyen, H., and Wu, M.C., 1999, “A HighSpeed Low-Voltage Stress-Induced Micromachined 2 x 2 Optical Switch,” IEEE Photonics Technology Letters, 11(11), pp. 1396-1398.

[2] Ma, X. and Kuo, G.S., 2003, "Optical Switching Technology Comparison: Optical MEMS vs. Other Technologies," IEEE Optical Communications, November, pp. S16-S23.

[3] Peter, Y.-A., Gonte, F., Herzig, H.P., and Dandliker, R., 2002, "Micro-Optical Fiber Switch for a Large Number of Interconnects Using a Deformable Mirror," IEEE Photonics Technology Letters, 14(3), pp. 301-303.

[4] Yeow, T.-W., Law, K.L.E., and Goldenberg, A., 2001, "MEMS Optical Switches," IEEE Communications Magazine, November, pp. 158-163.

[5] De Dobbelaere, P., Falta, K., Fan, L., Floechner, S., and Patra, S., 2002, "Digital MEMS for Optical Switching," IEEE Communications Magazine, March, pp. 88-95.

[6] Chen, C. and Lee, C., 2004, "Design and Modeling for Comb Drive Actuator with Enlarged Static Displacement," Sensors and Actuation A: Physical, 115, pp. 530-539. 
[7] Grade, J.D., Jerman, H., and Kenny, T.W., 2003, "Design of Large Deflection Electrostatic Actuators," Journal of Microelectromechanical Systems, 12(3), pp. 335-343.

[8] Piyabongkarn, D., Sun, Y., Rajamani, R., Sezen, A., and Nelson, B.J., 2005, "Travel Range Extension of a MEMS Electrostatic Microactuator," IEEE Transactions on Control Systems Technology, 13(1), pp. 138-145.

[9] Borovic, B., Hong, C., Liu, A.Q., Xie, L., and Lewis, F.L., 2004, "Control of a MEMS Optical Switch," $43^{\text {rd }}$ IEEE Conference on Decision and Control, pp. 3039-3044.

[10] Legtenberg, R., Groeneveld, A.W., and Elwenspoek, M., 1996, "Comb-Drive Actuators for Large Displacements," Journal of Micromechanics and Microengineering, 6, pp. 320329.

[11] Kamiya, D., Hayama, T., and Horie, M., 1999, "Electrostatic Comb-Drive Actuators Made of Polyimide for Actuating Micromotion Convert Mechanisms," Microsystem Technologies, 5, pp. 161-165.

[12] Zhou, G. and Dowd, P., 2003, “Tilted Folded-Beam Suspension for Extending the Stable Travel Range of CombDrive Actuators," Journal of Micromechanics and Microengineering, 13, pp. 178-183.

[13] Zhao, Y. and Cui, T., 2003, "Fabrication of HighAspect-Ratio Polymer-Based Electrostatic Comb Drives Using the Hot Embossing Technique,” Journal of Micromechanics and Microengineering, 13, pp. 430-435.

[14] Nanofabrication Center, accessed 12/3/2009, "NFC Equipment and Process List (http://www.nfc.umn.edu/equipment/)," University of Minnesota - Twin Cities, Minneapolis, MN.

[15] Madou, M.J., 2002, Fundamentals of Microfabrication: The Science of Miniaturization, CRC Press, Boca Raton, FL.

[16] Borovic, B., Lewis, F.L., Liu, A.Q., Kolesar, E.S., and Popa, D., 2006, "The Lateral Instability Problem in Electrostatic Comb Drive Actuators: Modeling and Feedback Control,” Journal of Micromechanics and Microengineering, 16, pp. 1233-1241.

[17] Huang, W. and Lu, G., 2004, "Analysis of Lateral Instability of In-Plane Comb Drive MEMS Actuators Based on a Two-Dimensional Model," Sensors and Actuators A: Physical, 113, pp. 78-85.

[18] Schonhardt, S., Korvink, J.G., Mohr, J., Hollenbach, U., and Wallrabe, U., 2009, "Optimization of an Electromagnetic Comb Drive Actuator," Sensors and Actuators A: Physical, 154(2), pp. 212-217.

[19] Juan, W.-H. and Pang, S.W., 1998, "High-AspectRatio Si Vertical Micromirror Arrays for Optical Switching," Journal of Microelectromechanical Systems, 7(2), pp. 207-213. 
ANNEX A

DISPLACEMENT SIMULATION CODE

\%\% Electrostatic Displacement Simulation

\% Author: DRB, 12/10/2009

clear;clc;

$\% \%$

$\%$ Material and Geometry Constants

$\%$

$\mathrm{E}=269 \mathrm{e}$;

epsilon $=8.85 \mathrm{e}-12$;

h_A $=20 \mathrm{e}-6$;

t_s $=3 e-6$;

$1=500 \mathrm{e}-6$;

$z=2 e-6$;

$\% \%$

$\%$ Initialize the tooth count and loop

through input voltage range.

$\%$

$\mathrm{n}=20$;

index $=1$;

for $V=0: 1: 150$

$v_{\_} p($ index $)=v$;

delta $=$

$\mathrm{n}^{*}$ epsilon* $1^{\wedge} 3^{*} \mathrm{~V}^{\wedge} 2 /\left(2{ }^{*} \mathrm{Z}^{*} \mathrm{E}^{*} \mathrm{t} \_\mathrm{S}^{\wedge} 3\right)$;

d_p $($ index $)=$ delta;

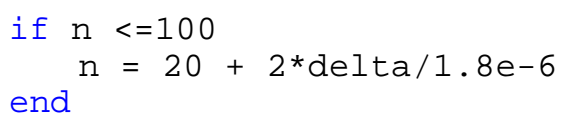

index $=$ index +1

end

$\% \%$

$\%$ output the results.

$\% \%$

$\% \%$

$\%$ Reset variables and calculate for constant tooth count.

$\%$

$\operatorname{plot}\left(\mathrm{V} \_\mathrm{p}, \mathrm{d} \_\mathrm{p}\right)$;

xlabel('Voltage (V)');

ylabel('Displacement (meters)');

hold on

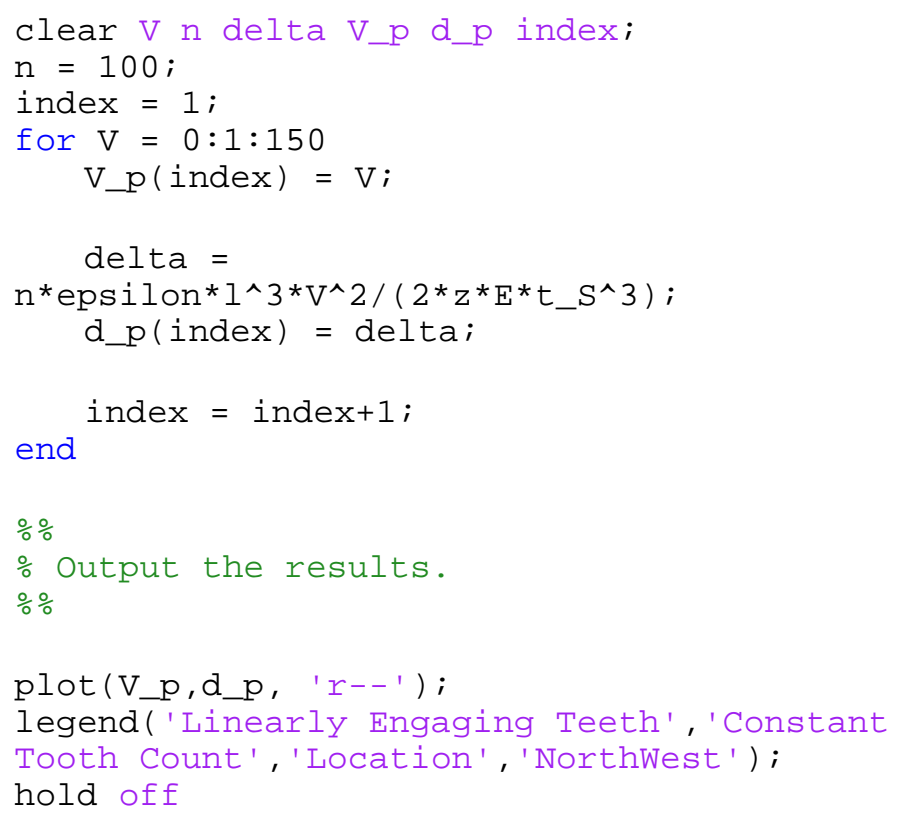

